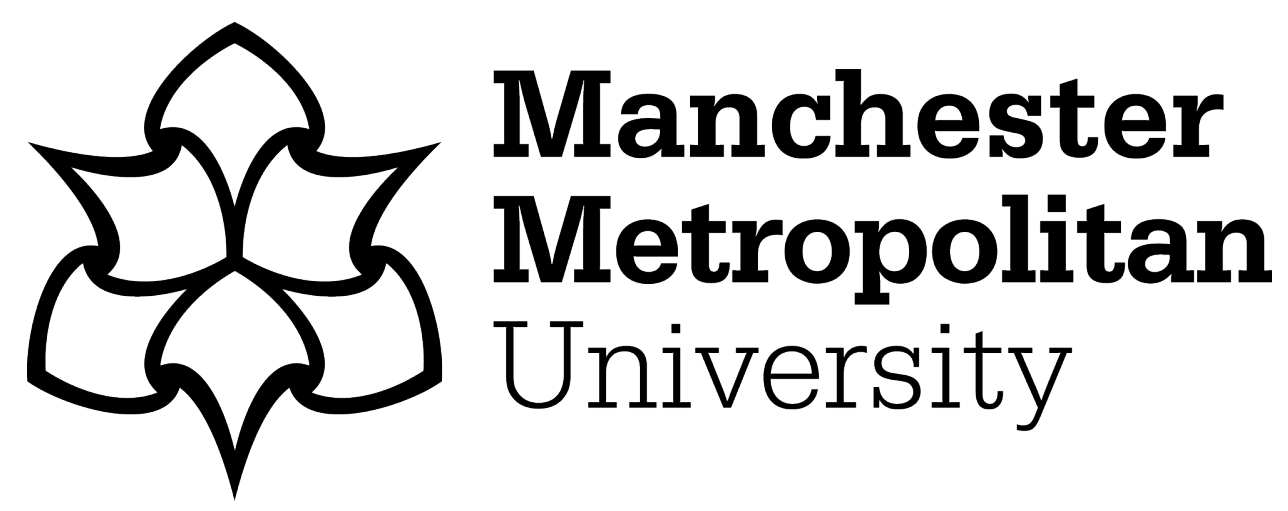

Moreland, Ethan, Borisov, Oleg V, Semenova, Ekaterina A, Larin, Andrey $\mathrm{K}$, Andryushchenko, Oleg N, Andryushchenko, Liliya B, Generozov, Edward V, Williams, Alun G and Ahmetov, Ildus I (2022) Polygenic Profile of Elite Strength Athletes. Journal of Strength and Conditioning Research, 36 (9). pp. 2509-2514. ISSN 1064-8011

Downloaded from: https://e-space.mmu.ac.uk/627102/

Version: Accepted Version

Publisher: Lippincott, Williams \& Wilkins

DOI: https://doi.org/10.1519/jsc.0000000000003901

Please cite the published version 


\section{Polygenic profile of elite strength athletes}

Running title: Genes for strength

Ethan Moreland ${ }^{1}$, Oleg V. Borisov ${ }^{2,3}$, Ekaterina A. Semenova ${ }^{2}$, Andrey K. Larin ${ }^{2}$, Oleg N. Andryushchenko $^{4}$, Liliya B. Andryushchenko ${ }^{5}$, Edward V. Generozov ${ }^{2}$, Alun G. Williams ${ }^{6,7}$, Ildus I. Ahmetov $1,5,8,9$

${ }^{1}$ Research Institute for Sport and Exercise Sciences, Liverpool John Moores University, Liverpool, United Kingdom

${ }^{2}$ Department of Molecular Biology and Genetics, Federal Research and Clinical Center of Physical-Chemical Medicine of Federal Medical Biological Agency, Moscow, Russia

${ }^{3}$ Institute for Genomic Statistics and Bioinformatics, University Hospital Bonn, Bonn, Germany

${ }^{4}$ Department of Physical Education, Financial University under the Government of the Russian Federation, Moscow, Russia

${ }^{5}$ Department of Physical Education, Plekhanov Russian University of Economics, Moscow, Russia

${ }^{6}$ Sports Genomics Laboratory, Department of Sport and Exercise Sciences, Manchester Metropolitan University, Manchester, United Kingdom

${ }^{7}$ Institute of Sport, Exercise and Health, University College London, London, United Kingdom

${ }^{8}$ Laboratory of Molecular Genetics, Kazan State Medical University, Kazan, Russia

${ }^{9}$ Sports Genetics Laboratory, St Petersburg Research Institute of Physical Culture, St Petersburg, Russia

Address correspondence to Ildus I. Ahmetov. Email: genoterra@mail.ru 


\section{ACKNOWLEDGEMENT}

We thank the Center for Precision Genome Editing and Genetic Technologies for Biomedicine, Federal Research and Clinical Center of Physical-Chemical Medicine of Federal Medical Biological Agency for providing computational resources for this project. 
Polygenic profile of elite strength athletes

Running title: Genes for strength 


\section{Abstract}

Strength is a heritable trait with unknown polygenic nature. So far, more than 200 DNA polymorphisms associated with strength/power phenotypes have been identified majorly involving non-athletic populations. The aim of the present study was to investigate individually and in combination the association of 217 DNA polymorphisms previously identified as markers for strength/power phenotypes with elite strength athlete status. A case-control study involved 83 Russian professional strength athletes (53 weightlifters, 30 powerlifters), 209 Russian and 503 European controls. Genotyping was conducted using micro-array analysis. Twenty-eight DNA polymorphisms (located near or in $A B H D 17 C, A C T G 1, A D C Y 3, A D P G K, A N G P T 2, A R P P 21$, BCDIN3D, CRTAC1, DHODH, GBE1, IGF1, IL6, ITPR1, KIF1B, LRPPRC, MMS22L, MTHFR, NPIPB6, PHACTR1, PLEKHB1, PPARG, PPARGC1A, R3HDM1, RASGRF1, RMC1, SLC39A8, TFAP2D, ZKSCAN5 genes) were identified to have an association with strength athlete status. Next, to assess the combined impact of all 28 DNA polymorphisms, all athletes were classified according to the number of 'strength' alleles they possessed. All highly elite strength athletes were carriers of at least 22 (up to 34) 'strength' alleles, while $27.8 \%$ of Russian controls had less than 22 'strength' alleles $(P<0.0001)$. The proportion of subjects with a high $(\geq 26)$ number of 'strength' alleles was significantly greater in highly elite strength athletes (84.8\%) compared to less successful strength athletes (64.9\%; OR=3.0, $P=0.042)$, Russian $(26.3 \%$; OR=15.6, $P<0.0001)$ or European $(37.8 \% ; \mathrm{OR}=6.4, P<0.0001)$ controls. This is the first study to demonstrate that the likelihood of becoming an elite strength athlete depends on the carriage of a high number of strength-related alleles.

Key words: strength performance, GWAS, DNA, genotype, polymorphism 


\section{Introduction}

It has long been established that skeletal muscle hypertrophy, hyperplasia, predominance of fasttwitch muscle fibers, improved neurological adaptation and high glycolytic capacity are major contributing factors to the performance of strength/power athletes $(9,10,33)$. Furthermore, strength athletes display completely different transcriptomic, biochemical, anthropometric, physiological, biomechanical and other characteristics compared to endurance athletes or nonathletic cohorts $(3,11,30,31)$. These differences can be explained both by environmental (training, nutrition, etc.) and genetic factors. Indeed, studies indicate that there is a strong heritability of power and strength-related traits with genetic factors accounting for $30-85 \%$ of the variation in isometric, isotonic and isokinetic strength, jumping ability, and other muscle strength phenotypes $(28,40)$.

Muscle strength/power phenotypes are accepted to be polygenic in nature - that is, multiple genetic factors influence the observed phenotype (20). For example, a polygenic profile (composed of variations of $A C E, A C T N 3, A G T, G D F 8, I L 6$, and NOS3 genes) was able to distinguish elite power athletes (Spanish jumpers, sprinters) from both endurance athletes and a nonathletic population (29). Power athletes (sprinters, speed-strength (i.e. jumpers, throwers etc.) and strength athletes (i.e. weightlifters and powerlifters)) demonstrate the highest level of these phenotypes of any population. A recent review provided evidence that at least 69 genetic markers are linked to elite power athlete status (23). Of those, 11 DNA polymorphisms (AGT rs699, ACTN3 rs1815739, CKM rs8111989, CNTFR rs41274853, GBF1 rs2273555, HIF1A rs11549465, MLN rs12055409, MTHFR rs1801131, PPARG rs1801282, PPARGC1A rs8192678 and ZNF608 rs4626333) have been shown to be associated with strength athlete status or strength performance in athletes $(2,5,8,12,13,14,19,27,39)$. These genes are implicated in skeletal muscle contraction (ACTN3), growth and development (AGT, MLN, ZNF608), glycolysis (HIF 1A), metabolism (GBF1, MTHFR, PPARG, PPARGC1A), energy homeostasis $(C K M)$, and neurogenesis $(C N T F R)$. 
As for the studies involving non-athletic cohorts, using a genome-wide association study (GWAS) approach, 196 DNA polymorphisms were associated with handgrip strength in three large GWASs. Specifically, the study conducted by Willems et al. of 195,180 white Europeans identified 16 single nucleotide polymorphisms (SNPs) near or within the genes involved in muscle structure and function, and associated them with handgrip strength (36). Matteini and coworkers examined associations of about 2.7 million SNPs in the GWAS with additional metaanalysis of individuals over 65 years old and reported 41 variants to be linked with handgrip strength (24). A more recent meta-analysis study by Tikkanen et al. (32) identified 139 loci associated with handgrip strength in a UK Biobank cohort. Interestingly, Tikkanen et al. (32) also observed a significant positive relationship between genes highly expressed in brain and genetic associations for grip strength. They also observed the most significant enrichment of differentially expressed genes in muscle with high proportion of genes implicated in the regulation of skeletal muscle contraction. Overall, studies involving power athletes and untrained subjects indicate that carriers of gene variants associated with increased muscle mass, high proportion of fast-twitch muscle fibers, improved anaerobic metabolism and neurological adaptation have greater strength potential and better chances to compete at the highest level.

One might hypothesize that multiple alleles associated with better power performance should be over-represented in elite strength athletes (weightlifters and powerlifters) compared to controls, thus playing some role in the selection of elite strength athletes. To date, no studies have attempted to quantify the impact of more than three genetic variants on elite strength performance / strength athlete status. The aim of the present study therefore was to investigate, individually and in combination, the association of 217 DNA polymorphisms previously identified as strength/power related phenotypes (195 SNPs associated with handgrip strength and 22 SNPs associated with power athlete status) with elite strength athlete status. 


\section{METHODS}

\section{Experimental Approach to the Problem}

To identify genetic markers associated with strength in 83 elite Russian strength athletes, we performed a case-control study using 217 SNPs previously discovered in non-athletic and athletic cohorts. Next, to assess the combined impact of all significant DNA polymorphisms, all athletes and controls were classified according to the number of 'strength' alleles they possessed. We then compared the proportion of subjects with a high number of 'strength' alleles between highly elite strength athletes, less successful strength athletes and controls.

\section{Subjects}

The study involved 83 strength athletes (53 weightlifters, 30 powerlifters; 54 men, 29 women). None of these athletes had ever tested positive for doping by a WADA-accredited laboratory. There were 46 athletes classified as 'highly elite' (ranked in the top 10 internationally; of those 36 were weightlifters) and 37 athletes classified as 'elite' (participants in international competitions, all national team members). Age, height and body mass of athletes are presented in Table 1. Controls were 209 healthy unrelated Russians (166 men and 43 women; $45.1 \pm 4.4$ yr) without any competitive sport experience (explored by survey). The athletes and controls were all Caucasians. In addition, data of European controls $(n=503)$ were used from the 1000 Genomes database for comparison with Russian athletes.

\section{Table 1 near here}

The overall study was approved by the Ethics Committee of the Physiological Section of the Russian National Committee for Biological Ethics. Written informed consent was obtained from each participant. The study complied with the guidelines set out in the Declaration of Helsinki and ethical standards in sport and exercise science research. The experimental 
procedures were conducted in accordance with the set of guiding principles for reporting the results of genetic association studies defined by the Strengthening the Reporting of Genetic Association studies (STREGA) Statement.

\section{Procedures}

Molecular genetic analysis in all Russian athletes and controls was performed with DNA samples obtained from leukocytes (venous blood), as previously described (26). Briefly, $4 \mathrm{ml}$ of venous blood was collected in tubes containing EDTA (Vacuette EDTA tubes, Greiner Bio-One, Austria). DNA extraction and purification were performed using a commercial kit according to the manufacturer's instructions (Technoclon, Russia). HumanOmnil-Quad BeadChips (Illumina Inc, USA) cover 1,140,419 SNPs including 217 of the 265 previously associated with relevant phenotypes in the literature (196 with handgrip strength, 69 with power athlete status), and were used for genotyping and subsequent imputation of the 217 available SNPs in athletes and controls (Supplemental Digital Content 1, contains information regarding all 217 SNPs). The assay required $200 \mathrm{ng}$ of DNA sample with a concentration of at least $50 \mathrm{ng} / \mu \mathrm{l}$.

\section{Statistical Analyses}

Statistical analyses were conducted using PLINK v1.90, R (version 3.4.3), and GraphPad InStat (GraphPad Software, Inc., USA) software. Haplotype phasing before imputation was performed using SHAPEIT. Imputation was performed using IMPUTE2. For phasing and imputation, we used 1000 Genomes Phase 3 data as a reference panel and imputed the variants with a frequency higher than $0.1 \%$ in the reference panel. Variants imputed with low certainty (info score $<0.6$ ) were filtered out after imputation. All SNPs that did not pass the Hardy-Weinberg equilibrium test were excluded from further analysis. All data are presented as mean (standard deviation). Genotype distribution and allele frequencies between athletes (and subgroups of athletes) and controls were compared using $\chi^{2}$ test. $P$ values $<0.05$ were considered statistically significant. 


\section{RESULTS}

Of the 217 SNPs, 28 (ABHD17C rs7165759 A, ACTG1 rs6565586 A, ADCY3 rs10203386 T, ADPGK rs4776614 C, ANGPT2 rs890022 A, ARPP21 rs1513475 C, BCDIN3D rs12367809 C, CRTAC1 rs563296 G, DHODH rs12599952 A, GBE1 rs9877408 A, IGF1 rs35767 A, IL6 rs1800795 G, ITPR1 rs901850 T, KIF1B rs11121542 G, LRPPRC rs10186876 A, MMS22L rs9320823 T, MTHFR rs1801131 G, NPIPB6 rs2726036 A, PHACTR1 rs6905419 C, PLEKHB1 rs7128512 G, PPARG rs1801282 G, PPARGC1A rs8192678 A, R3HDM1 rs6759321 T, RASGRF1 rs1521624 A, RMC1 rs303760 C, SLC39A8 rs13135092 A, TFAP2D rs56068671 T, ZKSCAN5 rs3843540 C) were nominally $(P<0.05)$ associated with strength athlete status using different models (additive, recessive or dominant) either in all strength athletes $(n=83)$ and / or subgroups of athletes (i.e. weightlifters $(n=53)$, powerlifters $(n=30)$, highly elite strength athletes $(\mathrm{n}=46)$, highly elite weightlifters $(\mathrm{n}=36))$ (Table 2). More details for each SNP are shown in Supplemental Digital Content 1. Although no association passed Bonferroni correction for multiple testing (i.e. $P$ value $=0.05 / 217$ SNPs $* 5$ groups $* 3$ models (additive, recessive, dominant $)=0.000015$ ), we felt justified to use 28 SNPs in the polygenic analysis given that we used SNPs already discovered independently, most via GWASs at genome-wide significance.

\section{Table 2 near here}

Next, to assess the combined impact of all 28 DNA polymorphisms, athletes and controls were classified according to the number of 'strength' alleles they possessed. All highly elite strength athletes were carriers of at least 22 (up to 34 ) 'strength' alleles, while $27.8 \%$ of Russian $(\mathrm{OR}=35.9, P<0.0001)$ and $17.9 \%$ of European $(\mathrm{OR}=20.4, P=0.0017)$ controls had less than 22 'strength' alleles. The proportion of subjects with a high $(\geq 26)$ number of 'strength' alleles was significantly greater in highly elite strength athletes (84.8\%) compared to less successful (elite) 
strength athletes $(64.9 \%$; $\mathrm{OR}=3.0, P=0.042)$, Russian $(26.3 \%$; $\mathrm{OR}=15.6, P<0.0001)$ or European (37.8\%; OR=6.4, $P<0.0001)$ controls (Figure 1). Furthermore, elite athletes also had greater proportion of subjects $(64.9 \%)$ with a high $(\geq 26)$ number of 'strength' alleles compared to Russian $(26.3 \%$; OR=5.2, $P<0.0001)$ or European $(37.8 \%$; OR=3.0, $P=0.0011)$ controls.

\section{Figure 1 near here}

\section{DISCUSSION}

To our knowledge, this is the first comprehensive study aimed to identify polygenic profile of strength athletes using more than three gene polymorphisms. To genotype and impute multiple DNA variants associated with power performance we used a micro-array analysis to identify 28 SNPs associated with elite strength athlete status in Russians. These SNPs are located in or near genes that have multiple functions including growth and development (ANGPT2, CRTAC1, IGF1, IL6, NPIPB6, R3HDM1, TFAP2D), metabolism (ABHD17C, ADCY3, ADPGK, BCDIN3D, DHODH, GBE1, ITPR1, LRPPRC, MTHFR, PPARG, PPARGC1A, RMC1, ZKSCAN5), cell motility (ACTG1, PHACTR1), neurogenesis (ARPP21, PLEKHB1, RASGRF1), DNA repair $(M M S 22 L)$ and intracellular transport $(K I F 1 B, S L C 39 A 8)$. More details of gene functions are presented in Supplemental Digital Content 1. Interestingly, of those 28 genes, 16 genes (ACTG1, ADCY3, ANGPT2, BCDIN3D, CRTAC1, GBE1, IGF1, KIF1B, LRPPRC, MMS22L, MTHFR, PHACTR1, PPARGC1A, R3HDM1, RASGRF1, ZKSCAN5) alter their expression in human skeletal muscle during adaptation to resistance training compared to pretraining and/or non-exercise and endurance training states (34). More details of gene expression during resistance training are shown in Supplemental Digital Content 1.

According to the GTEx portal, 22 SNPs (ABHD17C rs7165759, ACTG1 rs6565586, ADCY3 rs10203386, ADPGK rs4776614, ARPP21 rs1513475, BCDIN3D rs12367809, CRTAC1 rs563296, DHODH rs12599952, GBE1 rs9877408, IL6 rs1800795, KIF1B rs11121542, LRPPRC 
rs10186876, MTHFR rs1801131, NPIPB6 rs2726036, PHACTR1 rs6905419, PLEKHB1 rs7128512, PPARG rs1801282, R3HDM1 rs6759321, RMC1 rs303760, SLC39A8 rs13135092, TFAP2D rs56068671, ZKSCAN5 rs3843540) are functional and influence expression of genes in various tissues, including skeletal muscle, nerves, blood and thyroid tissue - all important in terms of physical performance and training adaptations. More details of gene function are provided in Supplemental Digital Content 1.

Next, using a panel of 28 SNPs, we identified that strength athletes possess at least 22 'strength' alleles, while $27.8 \%$ of Russian and $17.9 \%$ of European controls had less than 22 'strength' alleles. On the other hand, we found that most highly elite strength athletes were carriers of at least 26 'strength' alleles. In the Russian and European populations there are only $26.3 \%$ and $37.8 \%$ of people with such a polygenic profile compared to $84.8 \%$ in highly elite strength athletes.

To date, the concept that strength performance is likely to be determined by the simultaneous presence of many advantageous genetic variants has only been addressed in principle (20) or in a mixed cohort of speed-strength athletes $(15,17,29)$ : few studies have yet sought to define or quantify the impact of multiple (i.e. more than two) genotype combinations that influence strength performance / strength athlete status and none have attempted this for more than three genetic variants $(1,5,13,14)$. We have thus addressed this issue, in a study focused on 217 DNA polymorphisms associated with strength/power phenotypes.

Our study does have limitations. First, extension to, and replication within groups of differing geographic ancestry is needed to translate these findings more broadly $(18,25,38)$. In general, less than $50 \%$ of findings can be replicated in subsequent studies. Indeed, we could confirm the association of just three SNPs (MTHFR rs1801131, PPARG rs1801282, PPARGC1A rs8192678) out of 11 previously associated with strength athlete status. However, besides casecontrol studies, genotype-phenotype studies should be performed to identify genetic markers for physical performance $(1,4,6,16,19,21)$. Second, none of the associations passed correction for 
multiple testing, but we felt justified to use 28 SNPs in the polygenic analysis given that we used SNPs already associated with relevant phenotypes in this validation phase. Of those 28 SNPs, 23 were initially found in GWASs, meaning that in the discovery phase (the original articles) these SNPs have passed correction for multiple testing at genome-wide significance $\left(P<5.0 \cdot 10^{-8}\right)$. The other 5 SNPs were derived from previous candidate gene studies and associated in at least two previous independent cohorts of athletes. It is common not to adjust for multiple comparisons in the validation phase to prevent the loss of potentially important findings $(7,37)$. Third, the lack of functional data relating to 28 DNA polymorphisms needs to be addressed with further transcriptomic, histological and physiological studies. Further, the association of polygenic profile with alterations in muscle function in response to training is advocated. In addition, our study is limited to 217 common polymorphisms which were primarily selected because of previously reported associations with various aspects of strength performance. We strongly suspect that many additional common polymorphisms, and probably rare mutations as well, will be shown to be associated with strength performance in due course. Thus, we suspect that the 28 polymorphisms we have used constitute only a small fraction of the genetic factors that influence human muscle strength. However, looking to the future, when hundreds or thousands of polymorphisms will be discovered that contribute to the variability in human muscle strength, the power of such information as a practical tool for sports coaches will emerge. Although these 28 polymorphisms have been associated with high levels of achievement in strength sports, we still believe that this is not of sufficient influence to be used in the selection of athletes (35). Currently, 'performance tests' (such as vertical jump, isometric mid-thigh pull and weightlifting performance) or traditional laboratory tests (such as isokinetic dynamometry and handgrip strength) are used to help identify young athletes with appropriate physiological potential, and to guide them into suitable training and competition. Such tests may, in the future, be augmented by assessment of polygenic profile. 
In conclusion, our findings confirm the polygenic nature of elite strength performance, a classic complex trait, and demonstrate that the likelihood of becoming an elite strength athlete depends on the number of strength-related alleles an individual possesses.

\section{PRACTICAL APPLICATIONS}

Our results highlight the relationship between a genetic profile derived from 28 polymorphisms and elite competitive strength performance. While many more genetic factors undoubtedly remain undiscovered, these 28 provide a basis on which future, more comprehensive, genetic assessments might augment systems of identifying and nurturing talent in elite strength sports.

\section{REFERENCES}

1. Ahmetov II, Gavrilov DN, Astratenkova IV, et al. The association of ACE, ACTN3 and PPARA gene variants with strength phenotypes in middle school-age children. $J$ Physiol Sci 63: 79-85, 2013.

2. Ahmetov II, Mozhayskaya IA, Lyubaeva EV, Vinogradova OL, Rogozkin VA. PPARG Gene polymorphism and locomotor activity in humans. Bull Exp Biol Med 146: 630-632, 2008.

3. Ahmetov II, Vinogradova OL, Williams AG. Gene polymorphisms and fiber-type composition of human skeletal muscle. Int J Sport Nutr Exerc Metab 22: 292-303, 2012.

4. Al-Khelaifi F, Yousri NA, Diboun I, et al. Genome-Wide Association Study Reveals a Novel Association Between MYBPC3 Gene Polymorphism, Endurance Athlete Status, Aerobic Capacity and Steroid Metabolism. Front Genet 11:595, 2020.

5. Ben-Zaken S, Eliakim A, Nemet D, Meckel Y. Genetic Variability Among Power Athletes: The Stronger vs. the Faster. J Strength Cond Res 33: 1505-1511, 2019.

6. Boulygina EA, Borisov OV, Valeeva EV, et al. Whole genome sequencing of elite athletes. Biol Sport 37:295-304, 2020. 
7. Duncan L, Shen H, Gelaye B, et al. Analysis of polygenic risk score usage and performance in diverse human populations. Nat Commun 10: 3328, 2019.

8. Fedotovskaya ON, Popov DV, Vinogradova OL, Akhmetov II. Association of MuscleSpecific Creatine Kinase (CKMM) Gene Polymorphism with Physical Performance of Athletes. Hum Physiol 38:89-93, 2012.

9. Folland JP, Williams AG. The adaptations to strength training: morphological and neurological contributions to increased strength. Sports Med 37: 145-168, 2007.

10. Fry AC, Schilling BK, Staron RS, Hagerman FC, Hikida RS, Thrush JT. Muscle fiber characteristics and performance correlates of male Olympic-style weightlifters. $J$ Strength Cond Res 17: 746-754, 2003.

11. Fuku N, Kumagai H, Ahmetov II. Genetics of muscle fiber composition. In: Sports, Exercise, and Nutritional Genomics: Current Status and Future Directions. D.Barh. and I. Ahmetov, eds. Academic Press, pp 295-314, 2019.

12. Gabbasov RT, Arkhipova AA, Borisova AV, et al. The HIF1A gene Pro582Ser polymorphism in Russian strength athletes. J Strength Cond Res 27: 2055-2058, 2013.

13. Gineviciene V, Jakaitiene A, Aksenov MO, et al. Association analysis of ACE, ACTN3 and PPARGC1A gene polymorphisms in two cohorts of European strength and power athletes. Biol Sport 33: 199-206, 2016.

14. Grishina EE, Zmijewski P, Semenova EA, et al. Three DNA Polymorphisms Previously Identified as Markers for Handgrip Strength Are Associated With Strength in Weightlifters and Muscle Fiber Hypertrophy. J Strength Cond Res 33:2602-2607, 2019.

15. Guilherme JPLF, Bertuzzi R, Lima-Silva AE, Pereira ADC, Lancha Junior AH. Analysis of sports-relevant polymorphisms in a large Brazilian cohort of top-level athletes. Ann Hum Genet 82: 254-264, 2018.

16. Guilherme JPLF, Egorova ES, Semenova EA, et al. The A-allele of the FTO Gene rs9939609 Polymorphism Is Associated With Decreased Proportion of Slow Oxidative 
Muscle Fibers and Over-represented in Heavier Athletes. J Strength Cond Res 33: 691$700,2019$.

17. Guilherme JPLF, Lancha AH Jr. Single Nucleotide Polymorphisms in Carnosinase Genes (CNDP1 and CNDP2) are Associated With Power Athletic Status. Int J Sport Nutr Exerc Metab 27: 533-542, 2017.

18. Guilherme JPLF, Semenova EA, Zempo H, et al. Are Genome-Wide Association Study Identified Single-Nucleotide Polymorphisms Associated With Sprint Athletic Status? A Replication Study With 3 Different Cohorts. Int J Sports Physiol Perform. 2020. doi: 10.1123/ijspp.2019-1032

19. Homma H, Kobatake N, Sekimoto Y, et al. Ciliary Neurotrophic Factor Receptor rs41274853 Polymorphism Is Associated With Weightlifting Performance in Japanese Weightlifters. J Strength Cond Res. 2020. doi: 10.1519/JSC.0000000000003750.

20. Hughes DC, Day SH, Ahmetov II, Williams AG. Genetics of muscle strength and power: polygenic profile similarity limits skeletal muscle performance. J Sports Sci 29: 1425$1434,2011$.

21. Kikuchi N, Tsuchiya Y, Nakazato K, Ishii N, Ochi E. Effects of the ACTN3 R577X Genotype on the Muscular Strength and Range of Motion Before and After Eccentric Contractions of the Elbow Flexors. Int J Sports Med 39: 148-153, 2018.

22. Kusić D, Connolly J, Kainulainen H, et al. Striated muscle-specific serine/threonineprotein kinase beta segregates with high versus low responsiveness to endurance exercise training. Physiol Genomics 52:35-46, 2020.

23. Maciejewska-Skrendo A, Sawczuk M, Cięszczyk P, Ahmetov II. Genes and Power Athlete Status. In: Sports, Exercise, and Nutritional Genomics: Current Status and Future Directions. D.Barh. and I. Ahmetov, eds. Academic Press, pp. 41-72, 2019.

24. Matteini AM, Tanaka T, Karasik D, et al. GWAS analysis of handgrip and lower body strength in older adults in the CHARGE consortium. Aging Cell 15: 792-800, 2016. 
25. Mustafina LJ, Naumov VA, Cieszczyk P, et al. AGTR2 gene polymorphism is associated with muscle fibre composition, athletic status and aerobic performance. Exp Physiol 99(8): 1042-52, 2014.

26. Pickering C, Suraci B, Semenova EA, et al. A genome-wide association study of sprint performance in elite youth football players. J Strength Cond Res 33: 2344-2351, 2019.

27. Roth SM, Walsh S, Liu D, et al. The ACTN3 R577X nonsense allele is under-represented in elite-level strength athletes. Eur J Hum Genet 16: 391-394, 2008.

28. Roth SM. Genetic aspects of skeletal muscle strength and mass with relevance to sarcopenia. Bonekey Rep 1:58, 2012.

29. Ruiz JR, Arteta D, Buxens A, et al. Can we identify a power-oriented polygenic profile? J Appl Physiol 108:561-566, 2010.

30. Stepto NK, Coffey VG, Carey AL, et al. Global gene expression in skeletal muscle from well-trained strength and endurance athletes. Med Sci Sports Exerc 41: 546-565, 2009.

31. Storey A, Smith HK. Unique aspects of competitive weightlifting: performance, training and physiology. Sports Med 42: 769-790, 2012.

32. Tikkanen E, Gustafsson S, Amar D, et al. Biological Insights Into Muscular Strength: Genetic Findings in the UK Biobank. Sci Rep 8: 6451, 2018.

33. Trappe S, Luden N, Minchev K, et al. Skeletal muscle signature of a champion sprint runner. J Appl Physiol 118:1460-1466, 2015.

34. Vissing K, Schjerling P. Simplified data access on human skeletal muscle transcriptome responses to differentiated exercise. Sci Data 1:140041, 2014.

35. Wang G, Tanaka M, Eynon N, et al. The Future of Genomic Research in Athletic Performance and Adaptation to Training. Med Sport Sci 61:55-67, 2016.

36. Willems SM, Wright DJ, Day FR, et al. Large-scale GWAS identifies multiple loci for hand grip strength providing biological insights into muscular fitness. Nat Com 8: 16015, 2017. 
37. Wood AR, Esko T, Yang J, Vedantam S, Pers TH, Gustafsson S, et al. Defining the role of common variation in the genomic and biological architecture of adult human height. Nat Genet 46: 1173-1186, 2014.

38. Yvert TP, Zempo H, Gabdrakhmanova LJ, et al. AGTR2 and sprint/power performance: a case-control replication study for rs11091046 polymorphism in two ethnicities. Biol Sport 35: 105-109, 2018.

39. Zarebska A, Ahmetov, II, Sawczyn S, et al. Association of the MTHFR 1298A $>$ C (rs 1801131) polymorphism with speed and strength sports in Russian and Polish athletes. J Sports Sci 32: 375-382, 2014.

40. Zempo H, Miyamoto-Mikami E, Kikuchi N, Fuku N, Miyachi M, Murakami H. Heritability estimates of muscle strength-related phenotypes: A systematic review and meta-analysis. Scand J Med Sci Sports 27: 1537-1546, 2017. 


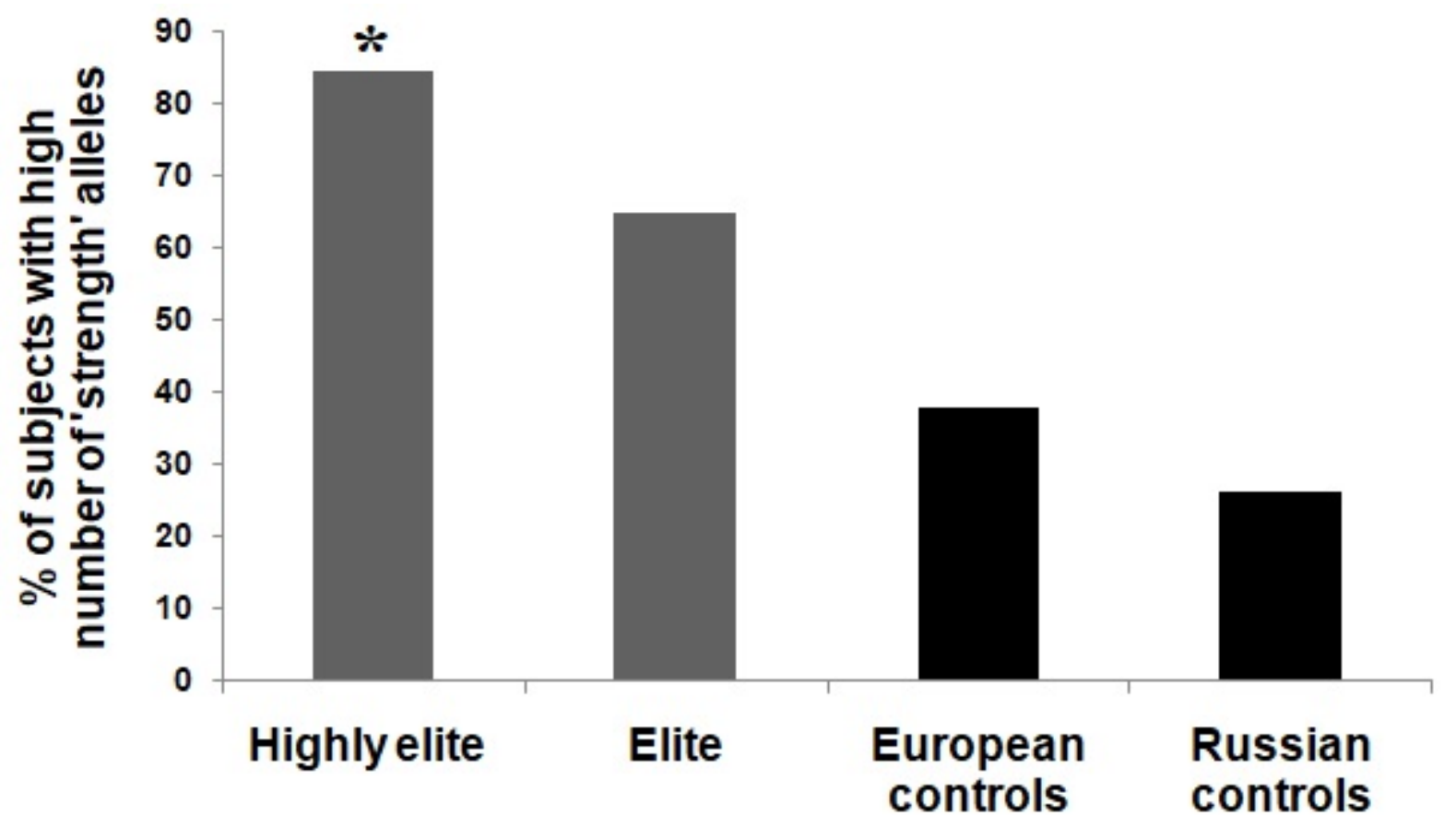

Figure 1. The combined impact of the 28 DNA polymorphisms. The percentage of subjects with a high $(\geq 26)$ number of 'strength' alleles is shown. *The proportion of subjects with a high number of 'strength' alleles was significantly greater in highly elite strength athletes $(84.8 \%)$ than elite strength athletes $(64.9 \%, P=0.042)$, Russian $(26.3 \%, P<0.0001)$ or European $(37.8 \%$, $P<0.0001)$ controls. 
Table 1. Anthropometric and performance variables in subjects from different groups

\begin{tabular}{|l|l|l|}
\hline \multirow{2}{*}{ Characteristics } & \multicolumn{2}{c|}{ Group } \\
\cline { 2 - 3 } & \multicolumn{1}{|c|}{ Weightlifters } & \multicolumn{1}{c|}{ Powerlifters } \\
\hline Males & $n=31$ & \\
\hline Age (years) & $23.7 \pm 0.7$ & $28.0 \pm 0.9$ \\
\hline Height (cm) & $179.0 \pm 1.6$ & $174.5 \pm 2.2$ \\
\hline Body mass (kg) & & $82.3 \pm 4.9$ \\
\hline Females & $96.7 \pm 3.7$ & \\
\hline Age (years) & & $n=7$ \\
\hline Height (cm) & $n=22$ & $25.0 \pm 0.7$ \\
\hline Body mass (kg) & $22.5 \pm 0.8$ & $160.7 \pm 2.6$ \\
\hline & & \\
\hline & $165.1 \pm 1.8$ & \\
\hline & & \\
\hline & & \\
\hline
\end{tabular}


Table 2. Associations between strength alleles identified in previous studies (in the same direction of association) and strength athlete status in the Russian groups of athletes and controls.

\begin{tabular}{|c|c|c|c|c|}
\hline \multirow[t]{2}{*}{ Strength allele } & \multirow[t]{2}{*}{ Group of athletes } & \multicolumn{2}{|c|}{$\begin{array}{l}\text { Frequency of the } \\
\text { strength allele, \% }\end{array}$} & \multirow[t]{2}{*}{$P^{*}$} \\
\hline & & Athletes & Controls & \\
\hline \multirow{2}{*}{$B C D I N 3 D$ rs12367809 C } & All strength athletes & 61.4 & \multirow{2}{*}{50.0} & 0.018 \\
\hline & Weightlifters & 61.5 & & 0.037 \\
\hline SLC39A8 rs13135092 A & All strength athletes & 97.5 & 93.0 & 0.043 \\
\hline \multirow{3}{*}{$R A S G R F 1$ rs1521624 A } & All strength athletes & 53.8 & \multirow{3}{*}{42.6} & 0.019 \\
\hline & Weightlifters & 52.9 & & 0.044 \\
\hline & Highly elite strength athletes & 54.4 & & 0.047 \\
\hline CRTAC1 rs563296 G & All strength athletes & 55.1 & 45.6 & 0.048 \\
\hline \multirow{4}{*}{$R 3 H D M 1$ rs6759321 T } & All strength athletes & 66.4 & \multirow{4}{*}{49.5} & 0.001 \\
\hline & Weightlifters & 68.5 & & 0.001 \\
\hline & Highly elite strength athletes & 63.8 & & 0.026 \\
\hline & Highly elite weightlifters & 71.0 & & 0.002 \\
\hline$A R P P 21$ rs1513475 C & Highly elite strength athletes & 31.5 & 23.7 & 0.039 \\
\hline$A D C Y 3 \mathrm{rs} 10203386 \mathrm{~T}$ & Highly elite strength athletes & 62.0 & 54.5 & 0.043 \\
\hline PHACTR1 rs6905419 C & Highly elite strength athletes & 85.6 & 72.4 & 0.01 \\
\hline \multirow{2}{*}{$M M S 22 L$ rs9320823 T } & Highly elite strength athletes & 43.3 & \multirow{2}{*}{30.3} & 0.025 \\
\hline & Highly elite weightlifters & 44.3 & & 0.027 \\
\hline C18orf8 rs303760 C & Highly elite strength athletes & 74.4 & 65.1 & 0.021 \\
\hline \multirow{2}{*}{$P P A R G$ rs $1801282 \mathrm{G}$} & Highly elite strength athletes & 26.1 & \multirow{2}{*}{15.6} & 0.022 \\
\hline & All strength athletes & 20.5 & & 0.045 \\
\hline TFAP2D rs56068671 T & Weightlifters & 10.8 & 4.6 & 0.03 \\
\hline PLEKHB1 rs7128512 G & Weightlifters & 95.3 & 88.7 & 0.046 \\
\hline GBE1 rs9877408 A & Weightlifters & 69.8 & 61.2 & 0.027 \\
\hline \multirow{4}{*}{ IL6 rs $1800795 \mathrm{G}$} & Weightlifters & 70.6 & \multirow{4}{*}{51.5} & 0.0005 \\
\hline & All strength athletes & 63.6 & & 0.009 \\
\hline & Highly elite strength athletes & 64.4 & & 0.0268 \\
\hline & Highly elite weightlifters & 70.0 & & 0.0042 \\
\hline MTHFR rs1801131 G & Weightlifters & 36.5 & 23.1 & 0.0079 \\
\hline
\end{tabular}




\begin{tabular}{|c|c|c|c|c|}
\hline & All strength athletes & 34.1 & & 0.008 \\
\hline & \begin{tabular}{|l|} 
Highly elite weightlifters \\
\end{tabular} & 37.5 & & 0.012 \\
\hline & Highly elite weightlifters & 54.2 & & 0.001 \\
\hline & All strength athletes & 45.5 & & 0.014 \\
\hline 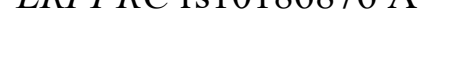 & Weightlifters & 45.3 & & 0.04 \\
\hline & Highly elite strength athletes & 50.0 & & 0.006 \\
\hline DHODH rs12599952 A & Highly elite weightlifters & 44.4 & 31.3 & 0.041 \\
\hline NPIPB6 rs2726036 A & Highly elite weightlifters & 68.6 & 56.4 & 0.013 \\
\hline & Highly elite weightlifters & 30.9 & & 0.022 \\
\hline ITPR1 rs901850 T & Weightlifters & 27.6 & 18.4 & 0.049 \\
\hline & Highly elite strength athletes & 26.7 & & 0.036 \\
\hline$A B H D 17 C$ rs $7165759 \mathrm{~A}$ & Powerlifters & 36.2 & 22.7 & 0.034 \\
\hline & Powerlifters & 44.4 & & 0.042 \\
\hline 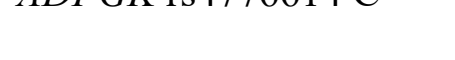 & Highly elite strength athletes & 39.8 & & 0.047 \\
\hline$A C T G 1$ rs6565586 A & Powerlifters & 42.6 & 27.0 & 0.024 \\
\hline$A N G P T 2$ rs890022 A & Powerlifters & 15.0 & 6.3 & 0.03 \\
\hline$K I F 1 B$ rs $11121542 \mathrm{G}$ & Powerlifters & 95.0 & 87.2 & 0.04 \\
\hline ZKSCAN5 rs3843540 C & Powerlifters & 22.4 & 13.2 & 0.04 \\
\hline$I G F 1 \mathrm{rs} 35767 \mathrm{~A}$ & Powerlifters & 30.0 & 17.5 & 0.033 \\
\hline$P P A R G C 1 A$ rs8192678 A & Powerlifters & 45.0 & 31.1 & 0.04 \\
\hline
\end{tabular}

$* P<0.05$, all differences between athletes and controls are statistically significant. More details are shown in Supplemental Digital Content 1). 